\title{
Tag SNP Polymorphism of CCL2 and Its Role in Clinical Tuberculosis in Han Chinese Pediatric Population
}

\author{
Wei-Xing Feng ${ }^{1}$, Igor Mokrousov ${ }^{2 *}$, Bin-Bin Wang ${ }^{3,4}$, Hugh Nelson ${ }^{5}$, Wei-Wei Jiao' ${ }^{1}$ Jing Wang ${ }^{3,4}$, Lin \\ Sun ${ }^{1}$, Si-Rui Zhou ${ }^{3,4}$, Jing Xiao ${ }^{1}$, Yi Gu ${ }^{1}$, Xi-Rong $\mathrm{Wu}^{1}{ }^{1}$, Xu Ma ${ }^{3,4}$, Adong Shen ${ }^{1 *}$
}

1 Beijing Pediatric Research Institute, Beijing Children's Hospital affiliated to Capital Medical University, Beijing, China, 2 Laboratory of Molecular Microbiology, St Petersburg Pasteur Institute, St Petersburg, Russia, $\mathbf{3}$ Graduate School, Peking Union Medical College, Beijing, China, 4 National Research Institute for Family Planning, Beijing, China, 5 International SOS Clinic, Shekou, Shenzhen, China

\begin{abstract}
Background: Chemokine (C-C motif) ligand 2 CCL2/MCP-1 is among the key signaling molecules of innate immunity; in particular, it is involved in recruitment of mononuclear and other cells in response to infection, including tuberculosis (TB) and is essential for granuloma formation.

Methodology/Principal Findings: We identified a tag SNP for the CCL2/MCP-1 gene (rs4586 C/T). In order to understand whether this SNP may serve to evaluate the contribution of the CCL2 gene to the expression of TB disease, we further analysed distribution of its alleles and genotypes in 301 TB cases versus 338 non-infected controls (all BCG vaccinated) representing a high-risk pediatric population of North China. In the male TB subgroup, the $C$ allele was identified in a higher rate $(P=0.045)$, and, acting dominantly, was found to be a risk factor for clinical TB $(P=0.029)$. Homozygous $\Pi T$ genotype was significantly associated with lower CSF mononuclear leukocyte (ML) counts in patients with tuberculous meningitis (TBM) $(P=0.001)$

Conclusions/Significance: The present study found an association of the CCL2 tag SNP rs4586 C allele and pediatric TB disease in males, suggesting that gender may affect the susceptibility to TB even in children. The association of homozygous TT genotype with decreased CSF mononuclear leukocyte (ML) count not only suggests a clinical significance of this SNP, but indicates its potential to assist in the clinical assessment of suspected TBM, where delay is critical and diagnosis is difficult.
\end{abstract}

Citation: Feng W-X, Mokrousov I, Wang B-B, Nelson H, Jiao W-W, et al. (2011) Tag SNP Polymorphism of CCL2 and Its Role in Clinical Tuberculosis in Han Chinese Pediatric Population. PLoS ONE 6(2): e14652. doi:10.1371/journal.pone.0014652

Editor: Olivier Neyrolles, Institut de Pharmacologie et de Biologie Structurale, France

Received June 14, 2010; Accepted January 14, 2011; Published February 4, 2011

Copyright: (c) 2011 Feng et al. This is an open-access article distributed under the terms of the Creative Commons Attribution License, which permits unrestricted use, distribution, and reproduction in any medium, provided the original author and source are credited.

Funding: This study was done in the Beijing Pediatric Research Institute, Beijing Children's Hospital affiliated to Capital Medical University, Beijing, China. It was partly supported by National Natural Science Foundation of China (grant No. 30672258). The funders had no role in study design, data collection and analysis, decision to publish, or preparation of the manuscript.

Competing Interests: Dr. Igor Mokrousov is a PLOS ONE Academic Editor. The authors declare that no other competing interests exist.

*E-mail: imokrousov@mail.ru (IM); shenad16@hotmail.com (AS)

\section{Introduction}

With 9 million new cases of tuberculosis (TB) worldwide in 2008 [1], the disease caused by Mycobacterium tuberculosis remains a topmost health problem in China with over 1.3 million new cases/ year and a prevalence as high as 194/100,000 in adults and 98/ 100,000 in children $[1,2]$. Although more than one-third of the world's population is potentially infected with $M$. tuberculosis, only $10 \%$ will develop the clinical disease over a lifetime [3]. Some genetic factors that may account for the development of TB disease after infection include (list not exhaustive): chemokine(C-C motif) ligand 2 (CCL2, other designation: MCP-1), genes encoding human leukocyte antigen type, purinergic receptor $\left(\mathrm{P}_{2} \mathrm{X}_{7}\right)$, the solute carrier family 11 member al protein (SLC11A1, formerly known as NRAMP1), and vitamin D3 receptor $[4,5,6,7,8,9,10]$ although the data may vary among different ethnic groups.

CCL2 belongs to the CC chemokines characterized by two adjacent cysteine residues close to the amino terminus. As with many other CG chemokines, the CCL2 gene is located on chromosome $17 q 11.2$ in humans which has been linked to susceptibility to tuberculosis and includes genes coding for several chemokines where CCL2 clusters together with the nitric oxide synthase 2A $(\mathcal{N O S} 2 A)$ and CCL3-5 genes $[11,12]$. The CCL2 chemokine is produced as a protein precursor containing signal peptide of 23 amino acids and a mature peptide of 76 amino acids $[13,14]$. The chemokine CCL2 is the most potent chemoattractant and activator for mononuclear leukocytes (MLs) and attracts CD4 and $\gamma \delta \mathrm{T}$ cells $[15,16,17]$, which are central components of the granulomatous response [18]. CCL2 can be released directly from infected cells and by forming a chemical gradient, escort sensitive MLs to the site of tissue injury [19]. Expression of the CCL2 gene may be found in many disorders characterized by ML infiltration [20]. CCL2 (MCP-1) plasma levels were significantly higher in $\mathrm{TB}$ adult patients of Mexican and Han Chinese ancestry who were carriers of -2518 CCL2 genotype GG [4,7]. GCL2 binds to CCR2 receptors and was shown to down-regulate the production of IL-12 and selectively inhibit the induction of the Thl immune response to Staphylococcus aureus $[21,22]$. The association of $-2518 \mathrm{G}$ allele with TB was found in North-East Asian (north China and Korea) [4,5,7], Zambian 
[23] and Central-South American (Mexico, Peru) populations $[7,8]$ whereas lack of such an association was found for South-East Asian (South China/Hong Kong) [18], South African Colored and Indian [24,25] populations. In contrast, the CCL2 -2518G allele was significantly associated with resistance to $\mathrm{TB}$ in Ghanaians [26].

Childhood TB represents an important and sensitive part of the burden of TB in the community. Compared to adults, children are much more likely to progress to $\mathrm{TB}$ disease after exposure, to develop disease more rapidly, and to develop asymptomatic extrapulmonary disease, disseminated (miliary) TB and tuberculous meningitis (TBM) [27,28,29,30,31]. It has been suggested that disseminated TB may reflect Mendelian predispositions in a fraction of children (3-30\% estimated by Bayesian statistics), whereas adult pulmonary $\mathrm{TB}$ seems to reflect a more complex genetic predisposition [32,33].

Unlike previous studies that focused on the CCL2 promoter SNPs, we hereby report a tag SNP for the entire CCL2 gene region. We further evaluated whether its polymorphism may serve to elucidate the contribution of the CCL2 gene to the expression of TB disease in a BCG-vaccinated pediatric population in North China. We also compared the CCL2 genotypes and counts of the MLs and polymorphonuclear (PMN) cells in the cerebrospinal fluid (CSF) in the TBM subgroup.

\section{Results}

\section{TB patients and non-infected controls}

The case group $(\mathrm{n}=301)$ included $105(34.9 \%)$ cases of active pulmonary TB (PTB), $196(65.1 \%)$ cases of extrapulmonary TB (EPTB), of which was $118(39.2 \%)$ cases of TBM. Severe extrapulmonary tuberculosis (SevTB) identified in $172(57.1 \%)$ patients included those with two or more noncontiguous sites, miliary mottling, or involvement of meningitis, pericardium, spinal, intestinal and splenic sites with or without lung involvement. The LNTB (Less severe extrapulmonary tuberculosis) group included $24(8 \%)$ patients with extra-pulmonary peripheral sites restricted to lymph nodes, peripheral joints, skin or pleural effusions without involvement of lung parenchyma. The control group included 338 pediatric surgical patients without history of tuberculosis. The demographic characteristics are explained in Table 1. All study subjects were BCG vaccinated at birth. The mean age was 5.9 years (SD, 4.8; range, 2 months-17 years) in the TB patients group. The mean age in control group $(\mathrm{n}=338)$ was 5.7 years (SD, 4.1 ; range, 3 months -16 years). The proportions of male were observed $61.8 \%$ in both groups. There were no significant differences between the groups for age and sex. The children aged less than 4 years old constituted $48.6 \%, 46.5 \%$, $16.7 \%$ in PTB, SevTB and LNTB groups, respectively.

All TB cases were classified according to the diagnostic standards of the American Thoracic Society (ATS) [34], the Pediatric tuberculosis clinical diagnosis standard in China [35] and WHO guidelines for disease severity classification for non-HIV related TB [36]. Patients were included in the study if they were positive by one or more of the following criteria: Tspot-TB test, Acid-Fast Bacilli Stain, culture, imaging, fibreoptic bronchoscopy (FOB) observation. The clinical records of patients were reviewed by two pediatricians with experience of TB in children (Table 2).

\section{Tagged-Haplotype and Linkage Disequilibrium (LD) analysis}

In the CCL2 region (chr17:29603409..29609331, from $3000 \mathrm{bp}$

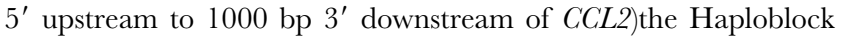
was constructed according to solid spine method and consisted of four SNPs (Fig. 1). These included two SNPs of promoter region rs1024611 (-2518A/G), rs1024610, one of exon 2 rs4586 and one of 3'UTR rs13900. Of this haplotype block, rs4586 was identified as the tag SNP for further association analysis. As the frequency of rs4586-C was similar to rs1024611-G, it was reasonable to expect that the rs4586-C and rs11024611-G (-2518G) alleles would be in perfect $\mathrm{LD}\left(\mathrm{D}^{\prime}=0.975 \mathrm{R}^{2}=0.9\right.$, based on China Han Beijing CHB dataset in HapMap PHASE 3: http://hapmap.ncbi.nlm.nih. gov/).

\section{Analysis of rs4586 variants versus disease phenotype and gender}

The genotypic and allelic frequencies of the tag SNP rs4586 of the CCL2 were analyzed in cases and controls. The genotypes were in HWE (Hardy-Weinberg equilibrium). The frequency of the $\mathrm{C}$ allele in TB patients was $61.3 \%$, whereas $\mathrm{T}$ allele frequency was $38.7 \%$; these frequencies did not differ significantly between cases and controls $(P=0.576)$. Analysis of genotypic distribution using $3 \times 2 \chi^{2}$ test revealed no significant difference between the two groups $(P=0.664)$. Moreover, no significant difference in the genotypic or allelic frequencies was found: (i) when control group was compared to different patient subgroups stratified by diagnosis; (ii) between PTB and EPTB subgroups (iii) between SevTB and LNTB subgroups (Table 3).

No significant differences between the TB and control groups were observed among male and female in genotypic frequencies (data not shown). At the same time, it was noted that $\mathrm{C}$ allele was more frequent in the male $\mathrm{TB}$ group $(2 \times 2$ test, $P=0.045$; $\mathrm{OR}=1.34 ; 95 \%$ CI 1.01-1.79). Different models of inheritance have been tested for combined sample and sex-stratified subsamples. In the male group, a statistical significance was observed when the $\mathrm{G}$ allele was regarded as a Mendelian dominant trait $(P=0.029$; OR $=1.94 ; 95 \%$ CI $1.06-3.56)$. In the female group no statistically significant differences were found (Table 4).

In genetic studies the power of a sample set to detect an association depends on the allele frequency and the size of its effect (estimated as OR). For complex infectious diseases, e.g., tuberculosis, a mild effect of many contributing genes is more expected; therefore we adopted the OR of 1.5. Accordingly, the power to detect association for the minor allele T of the tag SNP in combined sample was found to be high $(95 \%)$. Indeed under the stratification analysis, the power was lower $(80 \%$ for males, $62 \%$ for females) and this was a limitation of this study. Considering the difficulties in recruiting children and the relatively smaller size of the total pediatric TB population (compared to adult $\mathrm{TB}$ ), the design of our study may be considered appropriate. Indeed, similar case-control studies previously described have enrolled an even smaller number of individuals [37,38,39].

\section{Analysis of genotype versus CSF cells counts in TBM cases}

The data on ML and PMN counts in CSF were available for 110 of 118 TBM patients. The MLs count was decreased in TT genotype as compared with CG $(P=0.044)$ and TC $(P=0.008)$ genotypes. However, no difference was observed between $\mathrm{CC}$ and TC $(P=0.833)$ in the MLs level (Fig. 2A). The effect of the CCL2 rs4586 minor allele $\mathrm{T}$ on the level of MLs in CSF was assessed using both dominant (CT+TT versus $\mathrm{CG}$ ) and recessive (TT versus $\mathrm{CC}+\mathrm{CT}$ ) models. This comparison revealed a statistical significance for recessive model for $\mathrm{T}$ allele $(P=0.001)$ (Fig. 2B). The same analysis found no significant association of either genotype/allele and the number of PMNs in CSF (data not shown). 
Table 1. Demographic characteristics of study population.

\begin{tabular}{|c|c|c|c|c|c|}
\hline \multirow[t]{2}{*}{ Characteristic* } & \multicolumn{4}{|c|}{ Number of TB patients* } & \multirow[t]{2}{*}{ Control $(n=338)$} \\
\hline & PTB $(n=105)$ & SevTB $(n=172)$ & LNTB $(n=24)$ & Total $(\mathbf{n}=\mathbf{3 0 1})$ & \\
\hline \multicolumn{6}{|l|}{ Gender } \\
\hline Male & $68(64.8)$ & $100(58.1)$ & $18(75.0)$ & $186(61.8)$ & 209(61.8) \\
\hline \multicolumn{6}{|l|}{ Age } \\
\hline Mean (SD), Y & $5.4(4.7)$ & $5.8(4.7)$ & $9.5(4.7)$ & $5.9(4.8)$ & $5.7(4.1)$ \\
\hline \multicolumn{6}{|l|}{ Age group, $Y$} \\
\hline$<4$ & $51(48.6)$ & $80(46.5)$ & $4(16.7)$ & 135(44.9) & 131(38.7) \\
\hline $4-6$ & $14(13.3)$ & $21(12.2)$ & $2(8.3)$ & $37(12.3)$ & $73(21.6)$ \\
\hline $7-9$ & $18(17.1)$ & $22(12.8)$ & $5(20.8)$ & $45(14.9)$ & $58(17.2)$ \\
\hline $10-12$ & $11(10.5)$ & $33(19.2)$ & $7(29.2)$ & $51(16.9)$ & $58(17.2)$ \\
\hline $13-17$ & $11(10.5)$ & $16(9.3)$ & $6(25.0)$ & $33(11.0)$ & $18(5.3)$ \\
\hline BCG Vaccinated & Yes & Yes & Yes & Yes & Yes \\
\hline
\end{tabular}

*PTB, pulmonary TB; SevTB, severe extrapulmonary TB; LNTB, Less severe extrapulmonary TB; TBM, TB meningitis.

doi:10.1371/journal.pone.0014652.t001

\section{Discussion}

CCL2 is essential for the recruitment of mononuclear cells and subsequent activation of $\mathrm{T}$ lymphocytes, mononuclear and polymorphonuclear cells at the site of infection [17]. Studies of knockout mice indicated a non-redundant role of CCL2 in modulating MLs infiltration during inflammation [40]. Its involvement in pathogenesis of TB and granulomatous response suggested that variation of the CCL2 gene might play a role in conferring susceptibility to or protection from TB. However, knockout-mice lacking CCL2 were not found to be more susceptible to intravenous infection with $M$. tuberculosis [41] and published studies on the CCL2 variation from different world regions produced somewhat controversial conclusions $[7,8,26]$.

In this study, knowledge-based approach and linkage analysis using tagged-haplotype SNP were used in detecting CCL2 genetic associations: we identified a single tag SNP for the entire CCL2 gene (rs4586) and studied its role in clinical TB in Han Chinese pediatric population. Many studies demonstrated that haplotype diversity can be reduced to a smaller set of "haplotype-tagging" SNPs (htSNPs) identifying the most common haplotypes $[42,43]$. Tag SNPs are useful in whole-genome SNP association studies in which hundreds of thousands of SNPs across the entire genome are genotyped since being representative SNPs in a genome region with high $\mathrm{LD}$, they make it possible to identify genetic variation without genotyping every SNP in a chromosomal region of interest $[44,45]$. Here, rs4586 was chosen as haplotype-tagging SNP in order to reduce genotyping requirements by eliminating redundancy in the information provided by the other SNPs in the extended CCL2 genome region.

Our data indicated that a large proportion of cases were severe extrapulmonary TB (SevTB). No statistically significant differences were observed between cases of PTB and cases of SevTB. A comparison of carriers of the rs4586 genotype CC between SevTB and LNTB shows an increased proportion of homozygous CG among patients with SevTB (36.6\% vs 29.1\%, Table 3). Although this difference is not significant (OR 1.4, 95\% CI 0.55-3.57), it provides an interesting clue. In Chinese Han population (Hapmap-CHB) rs4586-C and -2518-G are in perfect LD (D'=0.975 $r^{2}=0.9$ ). Consequently, we speculate that the -2518 CCL2 genotype GG may be associated with a more severe disease in a pediatric population of Han Chinese as well as in Koreans, Mexicans and Peruvians [4,5,7,8] but not in non-Caucasian Africans, Indians and Caucasians $[24,25,26]$. Our analysis has a number of limitations (small sample size of the LNTB subgroup, difference in the mean age between SevTB and LNTB groups, 5.75 vs 9.45 years old, respectively) hence a larger study is needed to validate these preliminary results.

The significant associations were observed when results were stratified by gender. The CC and CT genotypes and the common $\mathrm{C}$ allele were significantly more prevalent in the male TB subgroup. As

Table 2. Diagnostic modality used for confirmation of TB.

\begin{tabular}{lll}
\hline & & \\
\hline Investigation or diagnostic procedure & Number of TB patients (positive/total)* & EPTB(n= 196) \\
\cline { 2 - 3 } & $\mathbf{P T B}(\mathbf{n}=\mathbf{1 0 5})$ & $169 / 196$ \\
\hline PPD & $100 / 105$ & $31 / 31$ \\
Tspot-TB test & $21 / 21$ & $12 / 196$ \\
Acid-Fast Bacilli Stain & $8 / 105$ & $22 / 196$ \\
Culture & $8 / 105$ & $155 / 196$ \\
Imaging & $105 / 105$ & $16 / 20$ \\
FOB Observation & $25 / 35$ &
\end{tabular}

*PTB, pulmonary TB; EPTB, extrapulmonary TB; FOB, fibreoptic bronchoscopy.

doi:10.1371/journal.pone.0014652.t002 


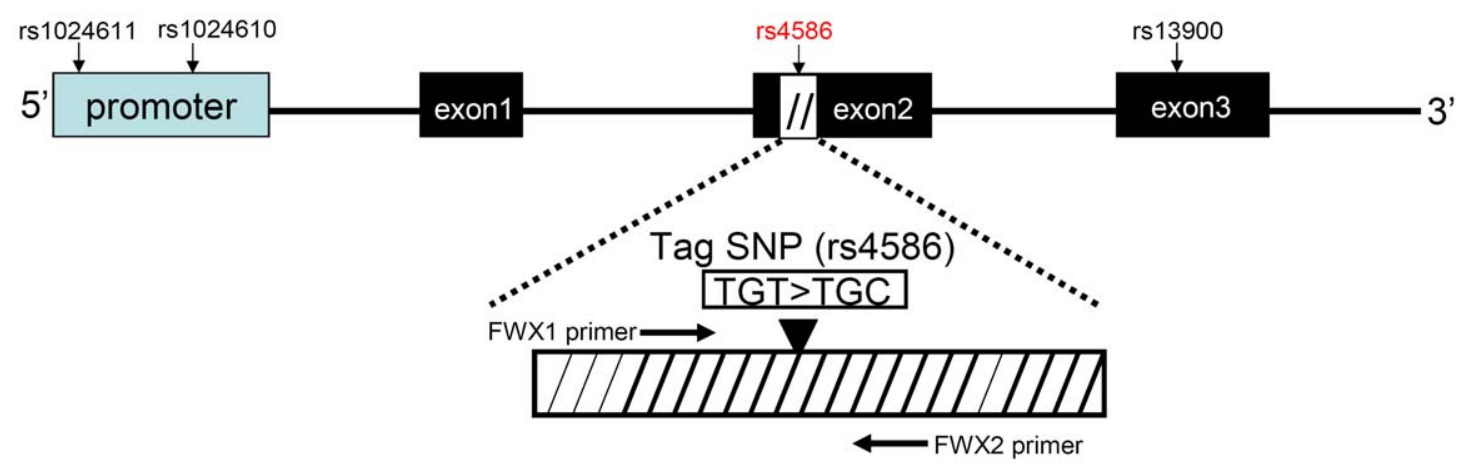

Figure 1. CCL2 gene structure and location of tag SNP rs4586 (T/C), a synonymous mutation at codon 35-Cys in exon 2 of CCL2. doi:10.1371/journal.pone.0014652.g001

rs4586-C was found to be associated with susceptibility to TB in males, we speculate that $-2518-\mathrm{G}$ may also be associated with TB in Chinese male children. This hypothesis should be verified in the further study focused specifically on the -2518 SNP. The prevalence of $\mathrm{TB}$ varies on the basis of sex that may be a modifier in TB development $[46,47]$. It is possible that a gender-related gene is involved in modification of TB development, and additional research must be performed to clarify the biological effects of gender in TB disease. However, as the $P$-value was at borderline significant level (0.045), a larger study will give better indications to the role of this polymorphism in susceptibility to TB in males.

Unfortunately, no population data on rs4586 in relation to clinical TB are available yet for other populations whereas CCL2 promoter SNP -2518 (rs1024611) has been extensively analyzed for its association with clinical TB. In this view, we extended our discussion to this latter SNP located inside the genome region under study and found to be in LD with rs4586 in our population. We would like to note that the association of -2518 with clinical TB observed in some but not all populations might reflect a genuine association owing to LD with another, putatively causal polymorphism in the adjacent CCL2 gene region. When comparing the linkage plot of Chinese CHB population to the plots of the three African populations, clear differences of $\mathrm{LD}$ patterns became evident (Fig. 3). This is in general agreement with the known distinct LD patterns that exist in African and diverse non-African populations. East Asian (Chinese and Japanese) populations had very similar patterns of $\mathrm{LD}$ (for the whole haplotype block of 4 SNPs). In contrast, the association of CCL2 rs4586-C was independent of that of $-2518 \mathrm{G}$ in all African populations. A moderate LD was found in the Caucasian (US residents of European ancestry and Italians), Gujarati Indians, and Mexicans populations (Fig. 3). Accordingly, different LD patterns between $2518 \mathrm{G}$ and a causal unidentified variant adjacent to CCL2 in different populations might explain the association with TB in some but not all populations. To sum up, the role of the CCL2 -2518 and likely rs4586) in TB disease may differ for different large settings, Africans versus East Asians versus Europeans.

It is worth mentioning that a strong association for $-362 \mathrm{C}$ allele (rs2857656) with protection against $\mathrm{TB}$ was shown in the Ghanaian population [26], a finding in clear disagreement with our results since this SNP is located well within our study region. Following the above discussion on -2518 SNP in different populations, it is not unlikely that this discrepancy may also well be explained by variation in the LD patterns in East Asians and Africans. The rs4586 is a useful tag-SNP in Han Chinese and perhaps may be useful in certain other ethnic groups.

Compared to the previous studies of CCL2 and human susceptibility to TB $[4,5,7,8,25,26]$, this study was distinctive in three ways. First, the targeted rs4586 was a CCL2 tag SNP thus permitting us to assess the entire gene and surrounding regions (+3000 bp in 5'UTR and +1000 bp 3'UTR). Second, we focused on pediatric TB that is known to be clinically and pathologically different from adult TB [33]. Third, the subjects in the study were BCG vaccinated. The studies in Mexico and Peru [8] also focused on BCG-vaccinated adult individuals, and they found that the CCL2 -2518 GG and MMP-1-1607 2G/2G increased the

Table 3. Effect of CCL2 rs4586 variants on disease phenotype.

\begin{tabular}{|c|c|c|c|c|c|c|c|}
\hline \multirow[t]{2}{*}{ Subjects $(n) *$} & \multicolumn{3}{|c|}{ Genotype, n (\%) } & \multirow[t]{2}{*}{$P$-value } & \multicolumn{2}{|l|}{ Allele } & \multirow[t]{2}{*}{$P$-value } \\
\hline & CC & TC & TT & & $C(\%)$ & $\mathbf{T}(\%)$ & \\
\hline Control (338) & $117(34.6)$ & $170(50.3)$ & $51(15.1)$ & - & $404(59.8)$ & $272(40.2)$ & - \\
\hline Case (301) & $106(35.2)$ & $157(52.2)$ & $38(12.6)$ & 0.664 & $369(61.3)$ & $233(38.7)$ & 0.576 \\
\hline PTB (105) & $36(34.3)$ & $57(54.3)$ & $12(11.4)$ & 0.605 & $129(61.4)$ & 81(38.6) & 0.667 \\
\hline EPTB (196) & 70(35.7) & $100(51.0)$ & $26(13.3)$ & 0.843 & $240(60.6)$ & $156(39.4)$ & 0.786 \\
\hline SevTB (172) & 63(36.6) & $85(49.4)$ & $24(14.0)$ & 0.883 & $211(61.3)$ & 133(38.7) & 0.627 \\
\hline LNTB (24) & $7(29.1)$ & $15(62.5)$ & $2(8.0)$ & 0.462 & $29(60.4)$ & 19(39.6) & 0.929 \\
\hline SevTB vs LNTB & - & - & - & 0.464 & - & - & 0.902 \\
\hline PTB vs EPTB & - & - & - & 0.834 & - & - & 0.843 \\
\hline
\end{tabular}

*PTB, pulmonary tuberculosis; EPTB, extrapulmonary; SevTB, severe extrapulmonary tuberculosis; LNTB, Less severe extrapulmonary tuberculosis.

$C$ is ancestral allele, $T$ - derived allele when compared to the sequence of the chimpanzee.

doi:10.1371/journal.pone.0014652.t003 
Table 4. Relationship between CCL2 rs4586 polymorphisms and pediatric tuberculosis (TB), stratified by sex.

\begin{tabular}{|c|c|c|c|c|c|c|}
\hline \multicolumn{2}{|c|}{ Alleles and genotypes stratified by sex } & \multirow{2}{*}{$\begin{array}{l}\text { TB } \\
243(65.3)\end{array}$} & \multirow{2}{*}{$\begin{array}{l}\text { Control } \\
244(58.4)\end{array}$} & \multirow{2}{*}{$\begin{array}{l}\chi^{2} \text {-value* } \\
4.02\end{array}$} & \multirow{2}{*}{$\begin{array}{l}P \text {-value } \\
0.045\end{array}$} & \multirow{2}{*}{\begin{tabular}{|l} 
OR (95\%CII) \\
$1.34(1.01-1.79)$
\end{tabular}} \\
\hline Male & C & & & & & \\
\hline & $T$ & $129(34.7)$ & $174(41.6)$ & & & \\
\hline \multirow[t]{2}{*}{ Female } & C & $126(54.8)$ & $160(62.0)$ & 2.62 & 0.105 & $1.35(0.94-1.93)$ \\
\hline & $\mathrm{T}$ & $104(45.2)$ & $98(38.0)$ & & & \\
\hline \multirow[t]{2}{*}{ Male } & $\mathrm{CC}+\mathrm{TC}$ & 168(90.3) & $173(82.8)$ & 4.75 & 0.029 & $1.94(1.06-3.56)$ \\
\hline & $\pi$ & $18(9.7)$ & $36(17.2)$ & & & \\
\hline \multirow[t]{2}{*}{ Female } & $\mathrm{CC}+\mathrm{TC}$ & $95(82.7)$ & $114(88.4)$ & 1.64 & 0.200 & $0.63(0.30-1.29)$ \\
\hline & $\pi$ & $20(17.3)$ & $15(11.6)$ & & & \\
\hline
\end{tabular}

*Genotype $\chi 2$ value for comparison of case and control groups in the genotype and allele frequencies.

doi:10.1371/journal.pone.0014652.t004

likelihood of progression to PTB. In their study all the controls were latently infected individuals with positive PPD test and did not develop TB disease for a period of more than 2 years after exposure. Differences in study populations (children in this study and adults in [8]) may explain these disparate results, particularly with respect to the criteria for selection of controls.

In this view, it is important to mention the limitations encountered during the design of the present study. A perfect study of susceptibility to TB should rely on the selection of the latently infected individuals as controls. Tuberculin Skin Test (TST) is useful to detect infected individuals for research purposes: Flores-Villanueva's group found that $99 \%$ of heavily exposed adults with a TST $>15 \mathrm{~mm}$ are also positive to a QuantiFeron-TB test in tube [8]. However a use of TST in BCG-vaccinated children in a high-prevalence country, such as China, is more than challenging. In China, immediate chemoprophylaxis/treatment is provided to all PPD-positive children in spite of the fact that BCG-vaccination may confound the diagnosis of infection. Even if the truly infected controls have been identified for the research purposes in this project, they would be treated and treatment/chemoprophylaxis might modify the course of infection regardless of the genetic profiles of the study subjects. Assuming equal exposure of both groups

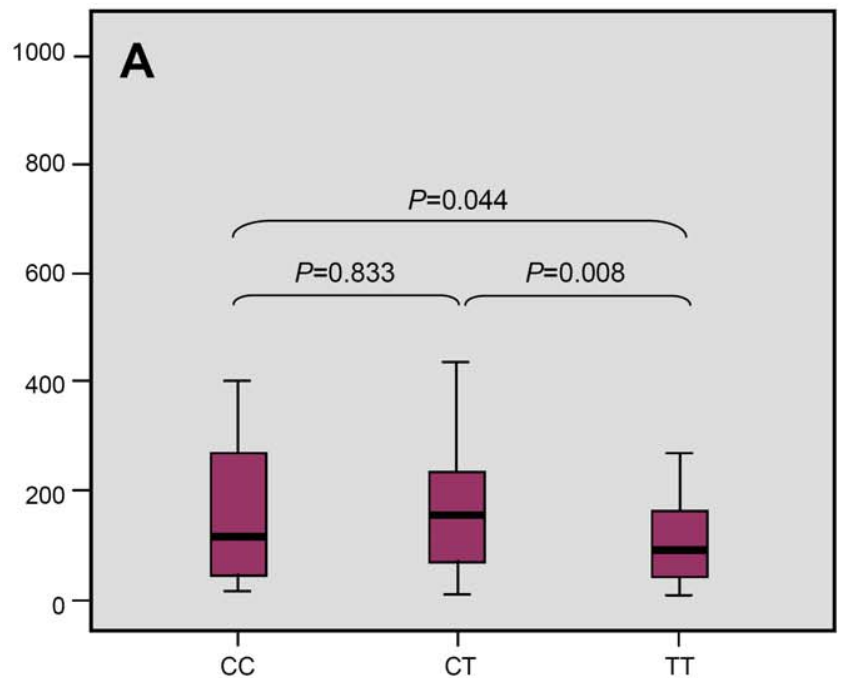

and since our controls remained uninfected during 2 years, we assumed "non-infected" status of the controls. In this case, we compared allele and genotype frequencies in tuberculosis patients with those non-infected controls, allowing us to distinguish susceptibility to infection from non-infection. An additional limitation of our study was that we did not have some detailed information for comparison between patients and controls, such as children's family history, habitat, family income, parents' educational level, family size. However, there is no logical reason why these differences should cause a specific SNP distribution to change, especially when the standard statistical precautions of measuring HWE and LD on other available SNP data are observed. Summing up, we believe that our decision to use non-infected children as controls was justifiable.

TB meningitis most commonly occurs in children aged $0-4$ years in the world areas with a high TB prevalence, such as China, and in adults in the areas with a low TB prevalence [48]. TB meningitis is the most severe complication of human M. tuberculosis infection causing death in $10 \%$ of cases with advanced disease and severe neurologic sequelae in $80 \%$ of survivors [49]. In this pediatric TB study, $39.2 \%$ (118/301) of cases were TBM patients, of which 110 cases were tested for MLs counts in CSF.

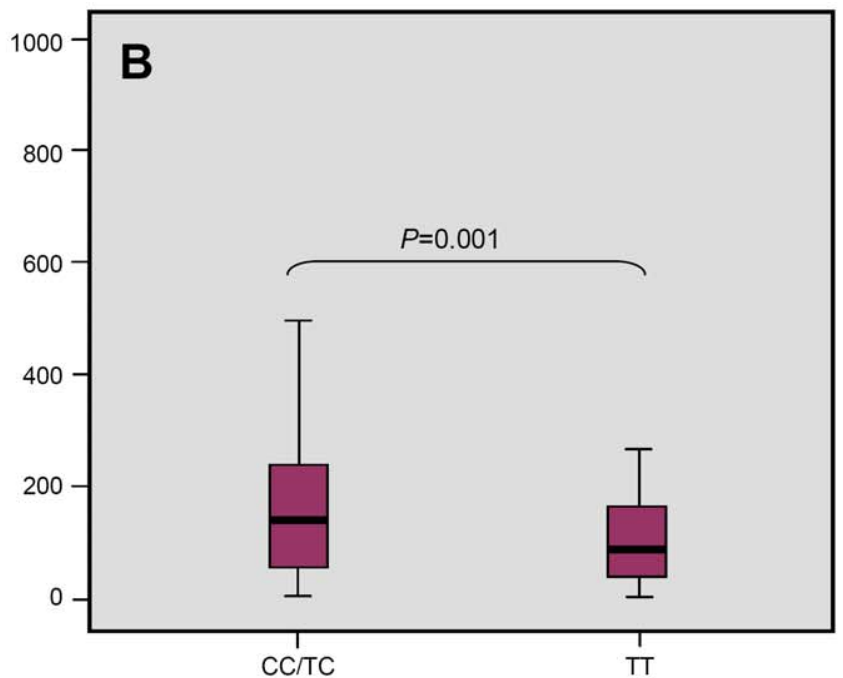

Figure 2. Numbers of MLs $\left(e^{6} / L\right)$ in CSF in different CCL2 tag SNP rs4586 genotype groups in TBM patients. (A) Comparison of homoand heterozygotes. (B) Testing dominant model for common allele C. doi:10.1371/journal.pone.0014652.g002 

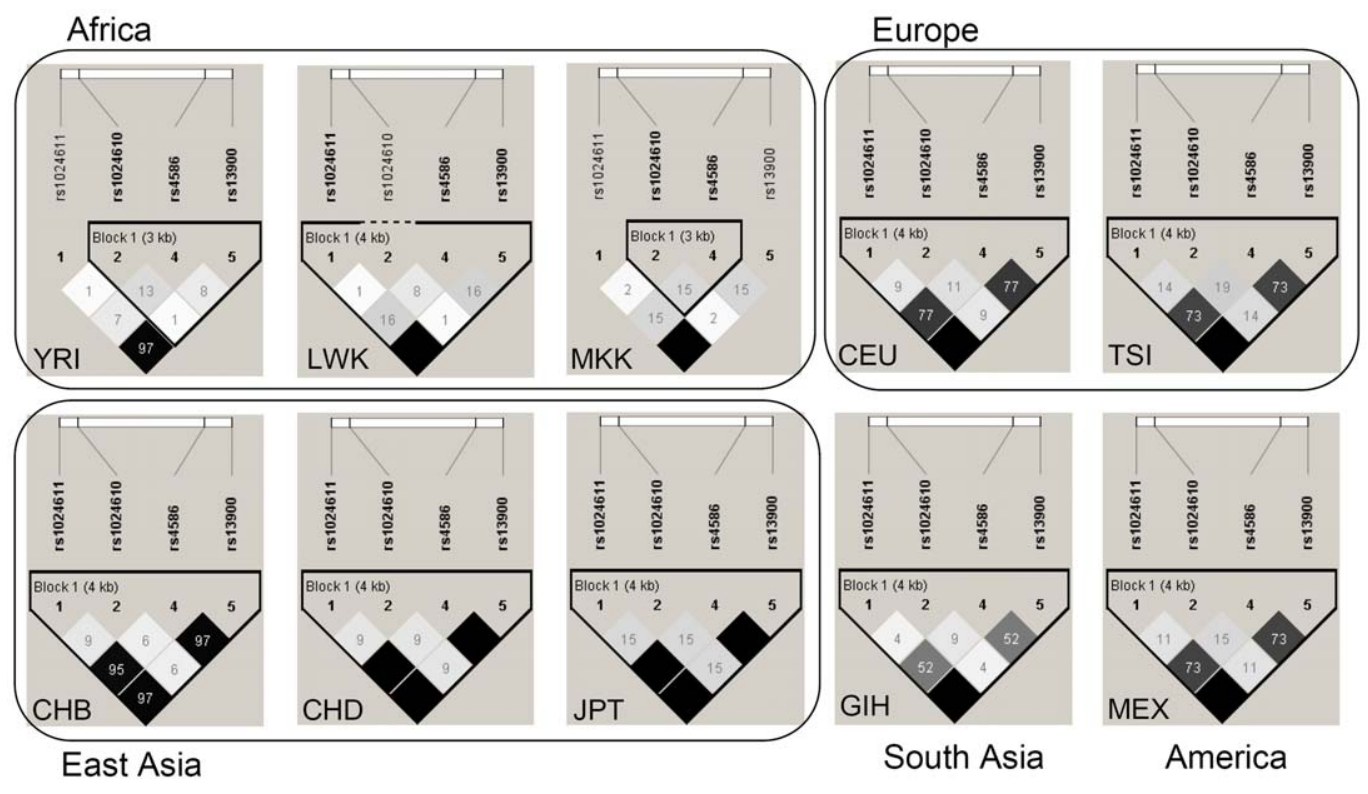

South Asia

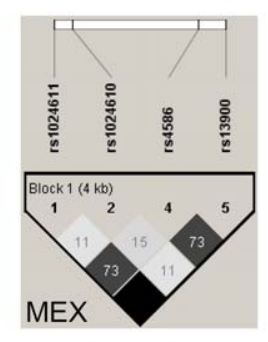

America

Figure 3. Pairwise LD $\left(r^{2}\right)$ plots of $C C L 2$ variants in different populations based on preliminary data extracted from the HapMap Phase 3 release. YRI, Yoruban in Ibadan, Nigeria; LWK, Luhya in Webuye, Kenya; MKK, Maasai in Kinyawa, Kenya; CEU, the US residents of northern and western European ancestry; TSI, Tuscan in Italy; CHB, Han Chinese in Beijing, China; CHD, Chinese in Metropolitan Denver, Colorado; JPT, Japanese in Tokyo, Japan; GIH, Gujarati Indians in Houston, Texas; MEX Mexican ancestry in Los Angeles, California.

doi:10.1371/journal.pone.0014652.g003

The appearance of MLs in the CSF is an important hallmark of TBM [50]. Chemokines and their receptors play a key role in directing the movement of MLs into the central nervous system (CNS) in pathological states [51]. CCL2 is essential for the recruitment of MLs hence the number of MLs in CSF may indeed be associated with CCL2 genotype. The MCP-1-deficient mouse study also indicated that CCL2 alone was responsible for mononuclear cell infiltration in several inflammatory models in vivo [41]. This prompted us to compare genotype versus phenotype, i.e., frequencies of the genotypes and alleles of the CCL2 tag SNP with the number of MLs (and PMNs) in the CSF. We found that subjects homozygous for $\mathrm{T}$ allele had decreased number of MLs in CSF (Fig. 2). Otherwise, the MLs level was higher than PMN level in CSF of TBM patients $(\mathrm{P}=0.02)$. The lack of association between CCL2 rs4586 genotypes and the number of the PMNs in the CSF is readily explained by the chemokine CCL2 more specific activity for MLs rather than PMNs.

However the mode of action of rs4586 polymorphism on the role of CCL2 in migration and attraction of MLs is unknown. Synonymous variations, in particular, are assumed to be functionally neutral both in clinical diagnosis and when measuring evolutionary distances between species. However, recent results suggested that silent mutations can have an effect on subsequent protein structure and activity [52,53]. Using the cystic fibrosis transmembrane conductance regulator (GFTR) exon 12 splicing as a model, it was demonstrated that $\sim 25 \%$ of synonymous variations result in exon skipping and, hence, in an inactive CFTR protein [52]. Furthermore, artificial combinations of CFTR exon 12 synonymous and non-synonymous substitutions were incompatible with normal RNA processing. The CCL2 tag SNP rs4586 (Cys35Cys) targeted in this study is located in exon 2 and may indeed affect the protein function at transcription control by inducing aberrant skipping of the mutant exon.
M. tuberculosis can cross the blood brain barrier (BBB) as a free (extracellular) organism or via infected MLs/PMNs [54]. The clinical manifestation of CNS tuberculosis is primarily a consequence of the inflammation which develops in response to M. tuberculosis in the CNS. Obstruction of the CSF by inflammatory infiltrate leads to hydrocephalus, and vasculitis contributes to infarction, causing potentially irreparable neurological damage [54]. Inhibition of this inflammation may therefore help in preventing the sequelae of CNS TB. Corticosteroids such as dexamethasone which suppress the production of inflammatory cytokines and chemokines lead to better outcomes and are recommended as adjunctive treatment for patients with TB meningitis $[55,56]$. Accordingly, patients with genotype TT with lower CSF MLs counts than genotypes CC/TC $(P=0.001)$ may cause less severe disease upon MLs infiltration. The TT genotype associated with lower level of MLs infiltration appears to act as a protection factor against stronger inflammation/more severe disease progression. If the TT genotype is associated with more efficient bacilli reduction in early stages of infection, then there may be evidence of decreased caseation in lungs, and possibly a lower incidence of inflammatory sequelae such as obstructive hydrocephalus after TBM, in TT homozygotes. Knowing that a patient has TT genotype may help in the correct clinical assessment of suspected TBM, by putting less reliance on an elevated ML count in the CSF, and greater reliance on other indicators.

An area for future study might be to see if the number of ventriculoperitonea $(\mathrm{VP})$ shunting procedures to prevent hydrocephalus is decreased in patients homozygous for TT allele. Lumbar puncture remains essential in the investigation of suspected meningitis. In this view, although the clinical significance of this SNP remains to be established, in practical terms it may be useful for simple, non-invasive, genotypic prediction of MLs low counts in CSF in different disorders. The knowledge that a patient has TT genotype will assist the 
clinician to maintain a high index of suspicion for TBM even when CSF ML count is low.

\section{Conclusion}

The present study found an association of the CCL2 tag SNP rs4586-C allele and pediatric TB disease in males, suggesting that the gender may affect the susceptibility to $\mathrm{TB}$ even in children. This study indirectly demonstrated that CCL2 may modulate the migration of MLs thereby influencing disease progression. The derived $\mathrm{T}$ allele, acting recessively, and $\mathrm{TT}$ genotype are associated with decreased level of MLs in CSF of TBM patients. The rs4586 SNP may be useful as a simple, non-invasive, genotypic marker able to predict low counts of MLs in CSF and aid in appropriate clinical assessment of suspected TBM.

\section{Materials and Methods}

\section{Ethics Statement}

The clinical investigation Tag SNP Polymorphism of CCL2 and its Role in Clinical Tuberculosis in Han Chinese Pediatric Population has been conducted according to the principles expressed in the Declaration of Helsinki. For research involving human participants, informed consent has been obtained from the patients or the guardian of the patients. The research has been approved by the Ethics Committee of the Beijing Children's Hospital.

\section{Study sample}

The data from 301 case patients and 338 control subjects were included in this study. All study individuals were of the Han Chinese ethnicity. All the cases and controls were vaccinated with BCG at birth, which was confirmed by the presence of a scar in the left shoulder and vaccination records in the clinical charts.

A total of 307 blood samples were obtained from pediatric patients with TB admitted at the Beijing Children's Hospital (affiliated with Capital Medical University; Beijing, China) between February 2005 and August 2010. Patients originated from Beijing city and surrounding provinces in North China. Children admitted consecutively to Beijing Children's hospital with clinical tuberculosis, a negative HIV test, were eligible to enter the study. In this study, primary immunodeficiency (PID) was ruled out by clinical history, examination, and the two tests for serum immunoglobulin levels (IgG, IgM and IgA) and lymphocyte subpopulations (CD3+ T-cells, CD56+ natural killer (NK) cells, CD19+ B-cells and also CD4+ and CD8+ T-cells measured by flow cytometry). Six patients were excluded due to PID. No patients born with a height and weight bellow the 50 percentile were included. None of patients had history of HIV infection, malnutrition, previous history of conditions affecting immune function, receipt of immunosuppressive therapy or other lung disease. In addition, all cases were new TB cases: none had a history of previous $\mathrm{TB}$ or had received previous treatment for TB.

Control group subjects were recruited from those admitted to the Beijing Children's Hospital between June 2005 and November 2007. Controls were matched with TB patients by age, sex, and ethnicity. The control group included 338 pediatric surgical patients without history of $\mathrm{TB}$, with normal radiographic examination findings, and with PPD skin test results $<5 \mathrm{~mm}$. The control group children were without the infection or the history of any inflammatory, autoimmune or infectious diseases, including TB and HIV infection. However, as we are dealing with a pediatric group, the possibility of a control to evolve as a case in future cannot be ruled out. All controls were therefore seen or questioned again 2 years after their initial visit, and their noninfection status was confirmed (with PPD skin test results $<5 \mathrm{~mm}$ ).
In the control groups, 50 randomly selected individuals were tested to determine whether they were infected with $M$. tuberculosis using Tspot-TB test (Oxford Immunotec, Abingdon, UK), and negative results were identified. A positive Tspot-TB test has a much higher sensitivity than skin testing as an indicator of active and latent $M$. tuberculosis infection $[57,58]$.

\section{DNA analysis and selection of haplotype-based tagging SNP}

Blood samples from children with $\mathrm{TB}$ and controls were collected and stored at $-20^{\circ} \mathrm{C}$. Genomic DNA was extracted from peripheral blood leukocytes according to standard methods.

We aimed to identify a set of SNPs that efficiently tag all of the common allelic variants and thus likely to tag most of the unknown common haplotypes in CCL2. The tag SNP was selected based on ability to tag surrounding variants (chr17:29603409..29609331) in the Han Chinese panel (Beijing, China) of the International HapMap project, NCBI build B36 assembly HapMap phase III (http://www.hapmap.org). Tagger software included in Haploview software 4.1 [59] was used to select tag SNP. The region used in tag SNP selection included a region from 3,000 bp 5 'upstream to 1000 bp 3 'downstream of CCL2 (Fig. 1). The synonymous TGG > TGT variation at codon 35-Cys (rs4586) was the only tag SNP in CCL2 obtained by aggressive tagging a minor allele frequency (MAF) of 0.15 , a pairwise correlation coefficient $r^{2}$ of 0.80 . LD was calculated and graphically displayed with the Haploview 4.2 software.

The rs4586 and its surrounding regions of CCL2 gene exon 2 were amplified with primers designed by Gene Tool V 1.0.0.1 (Launcher Program for Bio Tools Inc. Applications), sense primer FWX1 5'-CCGTGGTGCTGATCATCTGGA-3', and antisense primer FWX2 5'-GGTGGAGAGTGATGTTGGGGTTC$3^{\prime}$ (Fig. 1). The cycling conditions were as follows: initial denaturation at $94^{\circ} \mathrm{C}$ for $3 \mathrm{~min} ; 35$ cycles of $94^{\circ} \mathrm{C}$ for $30 \mathrm{~s}$, $58.9^{\circ} \mathrm{C}$ for $30 \mathrm{~s}$, and $72^{\circ} \mathrm{C}$ for $30 \mathrm{~s}$; and final elongation at $72^{\circ} \mathrm{C}$ for $10 \mathrm{~min}$. PCR products were directly sequenced using Big Dye Terminator 3.1 chemistry (Applied Biosystems Inc.) with the above primers and run on a 3730xl DNA analyzer (ABI).

\section{Determination of MLs and PMNs in CSF}

The Five or more lumbar punctures were obtained from children with tuberculous meningitis (TBM). Mononuclear leukocytes (MLs) and polymorphonuclear (PMN) cells in CSF were counted in a hemocytometer under a $100 \times$ oil immersion objective. Mononuclear leukocytes (MLs) are white blood cells with a one-lobed nucleus; two common forms of ML are monocytes and lymphocytes. Neutrophils together with basophils and eosinophils form part of the polymorphonuclear cell family (PMNs). The numbers were expressed as cells per cubic millimeter in undiluted CSF; the final value used in our analysis was the maximum value of all repetitions.

\section{Genetic and statistic analysis}

Statistical analysis was carried out using the Statistical Package for Social Sciences version 13.0 (SPSS, Chicago, IL, USA). Differences between non contiguous variables, genotype distribution and allele frequency were tested by chi-square analysis. The student's $t$-test was used to compare the numbers of the cells in the CSF between two genotypes, and ANOVA (Games-Howell test) was used to compare the numbers of cells in CSF for multiple comparisons. Significant differences were indicated by a $P$ value $<0.05$. Power calculation was performed with Quanto 1.2.4 software (http://hydra.usc.edu/gxe). LDs were calculated and graphically displayed with the Haploview 4.2 software. 


\section{Acknowledgments}

We are grateful to Dr. Pedro O. Flores-Villanueva for helpful comments that permitted us to significantly improve the manuscript. The authors thank all subjects for the ongoing participation in this study.

\section{References}

1. World Health Organization (2009) Global tuberculosis control: a short update to the 2009 report. Geneva: World Health Organization.

2. Jiang ZF, Yi ZW, Zhao SY (2006) Practical Pediatric Tuberculosis. Beijing: People's Medical Publishing House. 431 p.

3. Dolin PJ, Raviglione MC, Kochi A (1994) Global tuberculosis incidence and mortality during 1990-2000. Bull World Health Organ 72: 213-220.

4. Xu ZE, Xie YY, Chen JH, Xing LL, Zhang AH, et al. (2009) [Monocyte chemotactic protein-1 gene polymorphism and monocyte chemotactic protein-1 expression in Chongqing Han children with tuberculosis]. Zhonghua Er Ke Za Zhi 47: 200-203.

5. Yang BF, Zhuang B, Li F, Zhang CZ, Song AQ (2009) [The relationship between monocyte chemoattactant protein-1 gene polymorphisms and the susceptibility to pulmonary tuberculosis]. Zhonghua Jie He He Hu Xi Za Zhi 32: $454-456$.

6. Bellamy R (2003) Susceptibility to mycobacterial infections: the importance of host genetics. Genes Immun 4: 4-11.

7. Flores-Villanueva PO, Ruiz-Morales JA, Song CH, Flores LM, Jo EK, et al. (2005) A functional promoter polymorphism in monocyte chemoattractant protein-1 is associated with increased susceptibility to pulmonary tuberculosis. J Exp Med 202: 1649-1658.

8. Ganachari M, Ruiz-Morales JA, Gomez de la Torre Pretell JC, Dinh J, Granados J, et al. Joint effect of MCP-1 genotype GG and MMP-1 genotype $2 \mathrm{G} / 2 \mathrm{G}$ increases the likelihood of developing pulmonary tuberculosis in BCGvaccinated individuals. PLoS One 5: e8881.

9. Moller M, Hoal EG. Current findings, challenges and novel approaches in human genetic susceptibility to tuberculosis. Tuberculosis (Edinb) 90: 71-83.

10. Sambasivan V, Murthy KJ, Reddy R, Vijayalakshimi V, Hasan Q. P2X7 gene polymorphisms and risk assessment for pulmonary tuberculosis in Asian Indians. Dis Markers 28: 43-48.

11. Furutani Y, Nomura H, Notake M, Oyamada Y, Fukui T, et al. (1989) Cloning and sequencing of the cDNA for human monocyte chemotactic and activating factor (MCAF). Biochem Biophys Res Commun 159: 249-255.

12. Jamieson SE, Miller EN, Black GF, Peacock CS, Cordell HJ, et al. (2004) Evidence for a cluster of genes on chromosome 17q11-q21 controlling susceptibility to tuberculosis and leprosy in Brazilians. Genes Immun 5: 46-57.

13. Xu LL, Warren MK, Rose WL, Gong W, Wang JM (1996) Human recombinant monocyte chemotactic protein and other C-C chemokines bind and induce directional migration of dendritic cells in vitro. J Leukoc Biol 60: 365-371.

14. Yoshimura T, Yuhki N, Moore SK, Appella E, Lerman MI, et al. (1989) Human monocyte chemoattractant protein-1 (MCP-1). Full-length cDNA cloning, expression in mitogen-stimulated blood mononuclear leukocytes, and sequence similarity to mouse competence gene JE. FEBS Lett 244: 487-493.

15. Baggiolini M, Dewald B, Moser B (1994) Interleukin-8 and related chemotactic cytokines-CXC and CC chemokines. Adv Immunol 55: 97-179.

16. Penido C, Vieira-de-Abreu A, Bozza MT, Castro-Faria-Neto HC, Bozza PT (2003) Role of monocyte chemotactic protein-1/CC chemokine ligand 2 on gamma delta $\mathrm{T}$ lymphocyte trafficking during inflammation induced by lipopolysaccharide or Mycobacterium bovis bacille Calmette-Guerin. J Immunol 171: 6788-6794.

17. Premack BA, Schall TJ (1996) Chemokine receptors: gateways to inflammation and infection. Nat Med 2: 1174-1178.

18. Chu SF, Tam GM, Wong HS, Kam KM, Lau YL, et al. (2007) Association between RANTES functional polymorphisms and tuberculosis in Hong Kong Chinese. Genes Immun 8: 475-479.

19. Serbina NV, Jia T, Hohl TM, Pamer EG (2008) Monocyte-mediated defense against microbial pathogens. Annu Rev Immunol 26: 421-452.

20. Banisor I, Leist TP, Kalman B (2005) Involvement of beta-chemokines in the development of inflammatory demyelination. J Neuroinflammation 2: 7.

21. Mehrabian M, Sparkes RS, Mohandas T, Fogelman AM, Lusis AJ (1991) Localization of monocyte chemotactic protein-1 gene (SCYA2) to human chromosome 17q11.2-q21.1. Genomics 9: 200-203.

22. Rovin BH, Lu L, Saxena R (1999) A novel polymorphism in the MCP-1 gene regulatory region that influences MCP-1 expression. Biochem Biophys Res Commun 259: 344-348.

23. Buijtels PC, van de Sande WW, Parkinson S, Petit PL, van der Sande MA, et al. (2008) Polymorphism in CG-chemokine ligand 2 associated with tuberculosis in Zambia. Int J Tuberc Lung Dis 12: 1485-1488.

24. Moller M, Nebel A, Valentonyte R, van Helden PD, Schreiber S, et al. (2009) Investigation of chromosome 17 candidate genes in susceptibility to TB in a South African population. Tuberculosis (Edinb) 89: 189-194.

25. Alagarasu K, Selvaraj P, Swaminathan S, Raghavan S, Narendran G, et al. (2009) CCR2, MCP-1, SDF-1a \& DC-SIGN gene polymorphisms in HIV-1 infected patients with \& without tuberculosis. Indian J Med Res 130: 444-450.

\section{Author Contributions}

Conceived and designed the experiments: WXF XM AS. Performed the experiments: WXF BBW WWJ JW LS SRZ JX YG XRW. Analyzed the data: WXF IM. Wrote the paper: WXF IM HN AS.

26. Thye T, Nejentsev S, Intemann CD, Browne EN, Chinbuah MA, et al. (2009) MCP-1 promoter variant $-362 \mathrm{C}$ associated with protection from pulmonary tuberculosis in Ghana, West Africa. Hum Mol Genet 18: 381-388.

27. Newton SM, Brent AJ, Anderson S, Whittaker E, Kampmann B (2008) Paediatric tuberculosis. Lancet Infect Dis 8: 498-510.

28. Rigouts L (2009) Clinical practice: diagnosis of childhood tuberculosis. Eur J Pediatr 168: 1285-1290.

29. van Rie A, Beyers N, Gie RP, Kunneke M, Zietsman L, et al. (1999) Childhood tuberculosis in an urban population in South Africa: burden and risk factor. Arch Dis Child 80: 433-437.

30. Lobato MN, Cummings K, Will D, Royce S (1998) Tuberculosis in children and adolescents: California, 1985 to 1995 . Pediatr Infect Dis J 17: 407-411.

31. Walls T, Shingadia D (2004) Global epidemiology of paediatric tuberculosis. J Infect 48: 13-22.

32. Casanova JL, Abel L (2007) Primary immunodeficiencies: a field in its infancy. Science 317: 617-619.

33. Alcais A, Fieschi C, Abel L, Casanova JL (2005) Tuberculosis in children and adults: two distinct genetic diseases. J Exp Med 202: 1617-1621.

34. Diagnostic Standards and Classification of Tuberculosis in Adults and Children (2000) This official statement of the American Thoracic Society and the Centers for Disease Control and Prevention was adopted by the ATS Board of Directors, July 1999. This statement was endorsed by the Council of the Infectious Disease Society of America, September 1999. Am J Respir Crit Care Med 161: 1376-1395.

35. Hu YM, Jiang ZF, Zhu FT (2002) Textbook of pediatrics. 7th ed. Beijing: People's Medical Publishing House. pp 970-1013.

36. Maher D, Chaulet P, Spinaci S, Harries A (1997) Treatment of tuberculosisguidelines for national programmes. World Health Organization. pp 1-78.

37. Hoal-Van Helden EG, Epstein J, Victor TC, Hon D, Lewis LA, et al. (1999) Mannose-binding protein $\mathrm{B}$ allele confers protection against tuberculous meningitis. Pediatr Res 45: 459-464.

38. Cosar H, Ozkinay F, Onay H, Bayram N, Bakiler AR, et al. (2008) Low levels of mannose-binding lectin confers protection against tuberculosis in Turkish children. Eur J Clin Microbiol Infect Dis 27: 1165-1169.

39. Onay H, Ekmekci AY, Durmaz B, Sayin E, Cosar H, et al. Interferon-gamma gene and interferon-gamma receptor-1 gene polymorphisms in children with tuberculosis from Turkey. Scand J Infect Dis 42: 39-42.

40. Charo IF, Ransohoff RM (2006) The many roles of chemokines and chemokine receptors in inflammation. N Engl J Med 354: 610-621.

41. Lu B, Rutledge BJ, Gu L, Fiorillo J, Lukacs NW, et al. (1998) Abnormalities in monocyte recruitment and cytokine expression in monocyte chemoattractant protein 1-deficient mice. J Exp Med 187: 601-608.

42. Johnson GC, Esposito L, Barratt BJ, Smith AN, Heward J, et al. (2001) Haplotype tagging for the identification of common disease genes. Nat Genet 29: 233-237.

43. Ke X, Cardon LR (2003) Efficient selective screening of haplotype tag SNPs. Bioinformatics 19: 287-288.

44. Peiffer DA, Gunderson KL (2009) Design of tag SNP whole genome genotyping arrays. Methods Mol Biol 529: 51-61.

45. Xu Z, Kaplan NL, Taylor JA (2007) Tag SNP selection for candidate gene association studies using HapMap and gene resequencing data. Eur J Hum Genet 15: 1063-1070.

46. Leung KH, Yip SP, Wong WS, Yiu LS, Chan KK, et al. (2007) Sex- and agedependent association of SLC11A1 polymorphisms with tuberculosis in Chinese: a case control study. BMC Infect Dis 7: 19.

47. Holmes CB, Hausler H, Nunn P (1998) A review of sex differences in the epidemiology of tuberculosis. Int J Tuberc Lung Dis 2: 96-104.

48. Thwaites G, Chau TT, Mai NT, Drobniewski F, McAdam K, et al. (2000) Tuberculous meningitis. J Neurol Neurosurg Psychiatry 68: 289-299.

49. Schoeman J, Wait J, Burger M, van Zyl F, Fertig G, et al. (2002) Long-term follow up of childhood tuberculous meningitis. Dev Med Child Neurol 44: $522-526$.

50. Kim SH, Cho OH, Park SJ, Lee EM, Kim MN, et al. Rapid diagnosis of tuberculous meningitis by $\mathrm{T}$ cell-based assays on peripheral blood and cerebrospinal fluid mononuclear cells. Clin Infect Dis 50: 1349-1358.

51. Rebenko-Moll NM, Liu L, Cardona A, Ransohoff RM (2006) Chemokines, mononuclear cells and the nervous system: heaven (or hell) is in the details. Curr Opin Immunol 18: 683-689.

52. Pagani F, Raponi M, Baralle FE (2005) Synonymous mutations in GFTR exon 12 affect splicing and are not neutral in evolution. Proc Natl Acad Sci U S A 102: 6368-6372

53. Carlini DB, Stephan W (2003) In vivo introduction of unpreferred synonymous codons into the Drosophila Adh gene results in reduced levels of ADH protein. Genetics 163: 239-243. 
54. Be NA, Kim KS, Bishai WR, Jain SK (2009) Pathogenesis of central nervous system tuberculosis. Curr Mol Med 9: 94-99.

55. Thwaites GE, Nguyen DB, Nguyen HD, Hoang TQ, Do TT, et al. (2004) Dexamethasone for the treatment of tuberculous meningitis in adolescents and adults. N Engl J Med 351: 1741-1751.

56. Rock RB, Hu S, Gekker G, Sheng WS, May B, et al. (2005) Mycobacterium tuberculosis-induced cytokine and chemokine expression by human microglia and astrocytes: effects of dexamethasone. J Infect Dis 192: 2054-2058.
57. Mazurek GH, Jereb J, Lobue P, Iademarco MF, Metchock B, et al. (2005) Guidelines for using the QuantiFERON-TB Gold test for detecting Mycobacterium tuberculosis infection, United States. MMWR Recomm Rep 54: 49-55.

58. Pai M, Riley LW, Colford JM, Jr. (2004) Interferon-gamma assays in the immunodiagnosis of tuberculosis: a systematic review. Lancet Infect Dis 4: 761-776.

59. Barrett JC, Fry B, Maller J, Daly MJ (2005) Haploview: analysis and visualization of LD and haplotype maps. Bioinformatics 21: 263-265. 